\title{
Massless String Theory Black Holes as Black Diholes and Quadruholes
}

\author{
Tomás Ortín* \\ CERN Theory Division, CH-1211, Genève 23, Switzerland
}

(Received 15 February 1996)

\begin{abstract}
Massless black holes can be understood as bound states of a (positive-mass) extreme $a=\sqrt{3}$ black hole and a singular object with opposite (i.e., negative) mass with vanishing Arnowitt-Deser-Misner (total) mass but nonvanishing gravitational field. Supersymmetric balance of forces is crucial for the existence of this kind of bound states and explains why the system does not move at the speed of light. We also explain how supersymmetry allows for negative mass as long as it is never isolated but in bound states of total non-negative mass. [S0031-9007(96)00195-0]
\end{abstract}

PACS numbers: 11.25.-w, 04.70.Dy, 11.30.Pb

String theory, besides elementary strings, describes many interesting pointlike or extended objects with unbroken supersymmetries and of solitonic nature. Due to supersymmetry, many properties of these objects can be reliably studied in the framework of the low-energy supergravity theory. Extreme black holes (BH's) are particularly interesting string theory objects and the most interesting (and mysterious) amongst them are perhaps the massless ones. Their existence was conjectured by Strominger in Ref. [1] in the context of type II string theory duality phase transitions near conifold points. Massless $\mathrm{BH}$ solutions of the low-energy heterotic string effective action were recently discovered in Ref. [2] (see also Refs. [3-5]). Some properties of these objects are as follows:

(1) Their (asymptotically flat) canonical metric

$$
d s^{2}=\left(1-\frac{D^{2}}{r^{2}}\right)^{-1 / 2} d t^{2}-\left(1-\frac{D^{2}}{r^{2}}\right)^{1 / 2} d \vec{x}^{2}
$$

is singular when $r=D$. The singularity is a curvature singularity and the area of spheres of radius $r$ goes to zero in that limit.

(2) This metric does not seem to be the extreme limit of any nonextreme $\mathrm{BH}$ metric. (All this really makes the name $\mathrm{BH}$ quite inappropriate for them but we will stick to it for the moment.)

(3) The expansion of the $g_{t t}$ component of the metric far away from the singularity where the gravitational field is weak is

$$
g_{t t}=1+\frac{D^{2}}{2} \frac{1}{r^{2}}+\frac{3 D^{4}}{8} \frac{1}{r^{4}}+\mathcal{O}\left(\frac{1}{r^{6}}\right) .
$$

The coefficient of the $\frac{1}{r}$ term is $-2 m$, where $m$ is the Arnowitt-Deser-Misner (ADM) mass. Then, the ADM mass of these objects is zero (hence the adjective massless). In this limit, $g_{t t} \sim 1+2 \Phi$, where $\Phi$ is the Newtonian gravitational potential. Therefore,

$$
\Phi \sim \frac{D^{2}}{4} \frac{1}{r^{2}}+\frac{3 D^{4}}{16} \frac{1}{r^{4}}
$$

and has weakly repulsive (instead of attractive) character when acting on usual test particles [4] (in fact, $\frac{\partial}{\partial r} g_{t t}<$ $0, r>|D|$ ). If the Newtonian approximation was valid near the singularity, we could immediately say that the repulsion grows without bound in its neighborhood.

(4) They do not seem to move at the speed of light. Usual objects with zero rest mass moving at the speed of light have positive total energy and nonzero threemomentum but the total energy of massless BH's (their ADM mass) is zero. On the other hand, this metric does not admit any lightlike Killing vector and we must conclude that the whole ADM four-momentum of these objects actually vanishes. It is then surprising how, with zero total energy and momentum, there is something instead of nothing.

(5) When they are rightly embedded in a supergravity theory, they have half of $N=2$ or $N=4$ supersymmetries unbroken and the low-energy solutions describing them are also exact solutions of string theory.

In Ref. [4], it was observed that the repulsive force that appears at a finite distance from these objects may be interpreted as a gravitational interaction with its massive core. This Letter is an investigation into the nature of the "massive core" of "massless BH's" for which we will propose a model.

We will start by establishing a heuristic analogy between the expansions Eqs. (2) and (3) and multipole expansions in electrostatics. If we were studying the field created by some charge distribution confined in a region of space and we had the above expansions for large $r$, we would immediately say that the charge distribution has no monopole moment; that is, the total charge is zero. However, this does not mean that there is no charge. It just means that there are as many positive as negative charges, but its number cannot be deduced from the monopole moment alone. The existence of terms of higher order in $\frac{1}{r}$ indicates that the number of positive or negative charges is not zero (that is why we have a nontrivial field).

The analogy ends here, because multipole momenta terms are not spherically symmetric. Let us consider, though, two charge distributions not confined into a region, with positive and negative charge, respectively, 
spherically symmetric and concentric, such that the total (finite) charges are equal (but opposite). (I am indebted to Jorge Russo for a helpful discussion in which he proposed this model to me.) If the falloff of charge density of both distributions is different, the net charge density $\rho(r)$ is different from zero everywhere but its integral over the whole space is zero. Then, the net charge contained in a sphere of radius $r$,

$$
Q(r)=\int_{S^{3}(r)} d^{3} x^{\prime} \rho\left(r^{\prime}\right),
$$

is a function of $r$ that goes to zero when $r$ goes to infinity. Applying Gauss' law one gets the following dependence on $r$ for the electric field:

$$
E(r) \sim Q(r) / r^{2} .
$$

Now, if, for instance, $Q(r) \sim \frac{1}{r}$, then $E(r) \sim \frac{1}{r^{3}}$ and the electrostatic potential $\varphi \sim \frac{1}{r^{2}}$. The $\frac{1}{r}$ term appears only when $Q(r) \sim Q_{0}+\cdots$ and there is a net charge in the whole space.

Then, zero total charge and nontriviality of the field are compatible with spherical symmetry if the charge distribution is spread over the whole space. This would imply for our massless BH's that they could be composed of two concentric "charge distributions" with opposite signs and vanishing total "charge." The charge of gravity is the energy and it is carried by the gravitational field itself. Then, it is not necessary to have the whole space filled with positive- and negative-mass matter. Comparing the potential Eq. (3) with the above electrostatic potential $\varphi$, we would identify the $-\frac{D^{2}}{4} \frac{1}{r}$ as the effective mass $m(r)$ at a distance $r$ from the $B H$, much in the same spirit of Ref. [4].

There are a number of difficulties with the above hypothesis. First, it is usually thought that negative and positive masses in interaction are unstable and always lead to massless objects moving at the speed of light: opposite masses repel each other, but a negative mass accelerates in direction contrary to the force and therefore it follows the positive mass. This system does have positive energy, though the interaction energy between the objects (the rest mass energies would cancel) and it is different from a massless BH.

On the other hand, if there was another interaction between these objects such that the resulting force on each of them is zero, there would be static configurations describing these two objects in equilibrium. Rest masses and interaction energies would cancel and we would have a massless (zero-energy) system at rest. Its decay into a massless system moving at the speed of light (i.e., with positive energy) cannot spontaneously occur. The existence of additional charges would also explain why there is no annihilation between positive and negative masses if the additional charges carried by the objects do not add up to zero.

The next difficulty would be producing the corresponding static metric. If a no-force condition holds, one can expect supersymmetry. The fact that one of the masses is negative is no obstacle for having supersymmetry as long as the total ADM mass is not. [ It is tempting to identify the different constants that appear in multi-BH solutions as the different masses of these objects. There is, though, no rigorous way to assign a value to the mass of each individual $\mathrm{BH}$. There is only one asymptotically flat region and only one ADM mass, the total mass, can be rigorously defined. One can study initial-data sets describing $N$ nonextreme BH's which are not in equilibrium and in them there are $N+1$ asymptotic regions and individual and total ADM masses can be defined (see, for instance, [6] and references therein). The masses turn out to be the mentioned constants plus interaction energy terms. These terms vanish for the static multi-BH solutions and then it is physically reasonable to identify the constants with the masses. We will make heuristic reasonings which will be justified by the results.] If the solution has enough unbroken supersymmetries, the solution should be stable, at least under static perturbations of the metric.

Then, we should look for supersymmetric, extreme, multi-BH solutions. The one we are interested in was found in Ref. [7] and further discussed in Ref. [8]. This solution was later rediscovered in Ref. [9] in the framework of the theory described by the following simple action:

$$
\begin{aligned}
S= & \int d x^{4} \sqrt{-g}\left\{-R-2\left[(\partial \phi)^{2}+(\partial \sigma)^{2}+(\partial \rho)^{2}\right]\right. \\
& +\frac{1}{4} e^{-2 \phi}\left[e^{-2(\sigma+\rho)}\left(F^{(1) 1}\right)^{2}+e^{-2(\sigma-\rho)}\left(F^{(1) 2}\right)^{2}\right. \\
& \left.\left.+e^{2(\sigma+\rho)}\left(F^{(2)}\right)^{2}+e^{2(\sigma-\rho)}\left(F^{(2)}{ }_{2}\right)^{2}\right]\right\} .
\end{aligned}
$$

(Here we follow the conventions and notation of Ref. [10].) This action is a truncation of the low-energy effective action of the heterotic string [11]. In particular, $\phi$ is the four-dimensional dilaton.

The solution is given in terms of four independent harmonic functions $H^{(1)}, K^{(1)}, H^{(2)}, K^{(2)}\left(\partial_{\underline{i}} \partial_{\underline{i}} H=\partial_{\underline{i}} \partial_{\underline{i}} K=\right.$ $0, i=1,2,3)$

$$
\begin{gathered}
d s^{2}=U^{-\frac{1}{2}} d t^{2}-U^{\frac{1}{2}} d \vec{x}^{2}, \quad U=H^{(1)} K^{(1)} H^{(2)} K^{(2)}, \\
e^{-4 \phi}=\frac{H^{(1)} H^{(2)}}{K^{(1)} K^{(2)}}, \quad e^{-4 \sigma}=\frac{H^{(1)} K^{(2)}}{H^{(2)} K^{(1)}}, \\
e^{-4 \rho}=\frac{H^{(1)} K^{(1)}}{H^{(2)} K^{(2)}}, \quad F^{(a) 1}{ }_{t \underline{i}}=c^{(a)} \partial_{\underline{i}} \frac{1}{H^{(1)}}, \\
\tilde{F}^{(a) 2}{ }_{t \underline{i}}=d^{(a)} \partial_{\underline{i}} \frac{1}{K^{(1)}}, \quad a=1,2,
\end{gathered}
$$

where $\left(c^{(a)}\right)^{2}=\left(d^{(a)}\right)^{2}=1$ and

$$
\begin{aligned}
& \tilde{F}^{(1) 2}=e^{-2(\phi+\sigma-\rho) *} F^{(1) 2}, \\
& \tilde{F}_{2}^{(2)}=e^{-2(\phi-\sigma+\rho) *} F^{(2)} 2,
\end{aligned}
$$

and ${ }^{*} F$ is the Hodge dual of $F$. Usually, the $H$ 's and $K$ 's are chosen to be strictly positive, that is, all the constants 
in

$$
H^{(a)}=1+\sum_{n} \frac{q_{n}^{(a)}}{\left|\vec{x}-\vec{x}_{n}\right|}, \quad K^{(a)}=1+\sum_{n} \frac{p_{n}^{(a)}}{\left|\vec{x}-\vec{x}_{n}\right|},
$$

are non-negative constants to avoid the occurrence of singularities in the metric, but for any positive or negative value of the constants one gets a solution, and solutions with some negative $q$ 's or $p$ 's are what we are after. Bearing this in mind, and following Ref. [9], let us consider, for simplicity, solutions of the form

$$
H^{(a)}=1+\frac{q_{a}}{\left|\vec{x}-\vec{x}_{1}\right|}, \quad K^{(a)}=1+\frac{p_{a}}{\left|\vec{x}-\vec{x}_{2}\right|} .
$$

When all the $q$ 's and p's but one vanish, the solution is an $a=\sqrt{3}$ extreme dilaton $\mathrm{BH}$ if the nonvanishing constant is positive. Then, if several constants are positive, one can consider that the above solutions describe as many $a=\sqrt{3}$ BH's in equilibrium. The ADM mass of the system is $m=\frac{1}{4}\left(q_{1}+p_{1}+q_{2}+p_{2}\right)$ and would be positive. When the coordinates of all the BH's coincide one gets $a=1,1 / \sqrt{3}, 0$ extreme dilation BH's (depending on how many constants vanish) and therefore the above solution, and the corresponding extreme dilaton BH's, can be thought of as describing the external field of a bound state of "elementary" $a=\sqrt{3}$ BH's [9].

If we now allow for negative constants, one immediately sees from the above mass formula that one could get solutions with $m$ zero or negative. We are interested in the former. They can be thought of as describing usual extreme $a=\sqrt{3}$ dilaton BH's in equilibrium amongst them and with some other objects with negative mass. (We stress again that there is no rigorous way of telling what the mass of each individual object is, although, physically, it is clear that there must be some negative mass.)

The simplest massless combination $q_{1}=-q_{2}=$ $q, p_{1}=p_{2}=0$ a $\mathrm{BH}-$ anti-BH pair or dihole. Here it is clear why we have something instead of nothing with zero energy. On the other hand, the Ricci scalar of a single $a=\sqrt{3}$ extreme $\mathrm{BH}$ with metric

$$
d s^{2}=\left(1+\frac{q}{r}\right)^{-1 / 2} d t^{2}-\left(1+\frac{q}{r}\right)^{1 / 2} d \vec{x}^{2}
$$

is

$$
R=-q^{2} / 2 r(q+r)^{3} .
$$

When $q$ (the mass) is positive, the singularity is at $r=0$. When $q$ is negative, the singularity is at $r=|q|$. We have the same amount of positive mass matter and negative mass matter placed, concentrically, at different points and the two corresponding charge distributions should cancel only at infinity and, therefore, all the arguments given above apply to this case. We should get a massless, nontrivial, pointlike object with vanishing
ADM mass when the two massive objects are placed at the same point, and, in fact, substituting the $H$ 's into the metric and placing both BH's in the same point we recover the massless $\mathrm{BH}$ metric (1) with $D=q$.

Following the same reasoning as in Ref. [9] we would conclude that the known massless BH's are the effective field of a bound state of a pair of objects with opposite masses, or a dihole.

Another simple massless combination is $q_{1}=-q_{2}=$ $q, p_{1}=-p_{2}=p$. If the two electric charges $\pm q$ are placed at the same point and the two magnetic charge $\pm p$ 's are placed together at a different point, the resulting solution describes two massless diholes in equilibrium

$$
\begin{aligned}
d s^{2}= & {\left[\left(1-\frac{q^{2}}{r_{1}^{2}}\right)\left(1-\frac{p^{2}}{r_{2}^{2}}\right)\right]^{-1 / 2} d t^{2} } \\
& -\left[\left(1-\frac{q^{2}}{r_{1}^{2}}\right)\left(1-\frac{p^{2}}{r_{2}^{2}}\right)\right]^{1 / 2} d \vec{x}^{2} .
\end{aligned}
$$

When the four charges are placed at the same point one gets a quadruhole. If $p=q$, its metric takes a very simple form

$$
d s^{2}=\left(1-\frac{q^{2}}{r^{2}}\right)^{-1} d t^{2}-\left(1-\frac{q^{2}}{r^{2}}\right) d \vec{x}^{2} .
$$

Although more massless solutions are possible, these are perhaps the most interesting ones, at least to prove our point.

In conclusion, we have exhibited massless extreme BH's solutions that can be considered as bound states of positive and negative-mass objects satisfying a noforce condition.

It is difficult to avoid identifying these massless BH's with those which, according to Strominger [1], become massless when a type II string theory compactified on a Calabi-Yau (CY) threefold is near a conifold singularity of the CY moduli space and which can, in some cases, condensate [12], giving rise to a phase transition. This has been proposed in Ref. [5]. However, we have seen that the massless BH's found in Ref. [2] are really composite objects and they do not correspond to one-particle, but to two-particle states. It could well be that Strominger's massless BH's are also two-particle states. The fact that the $n_{1}=1$ BH's carries minimal $Z^{1}$ charge may not be an obstacle for this. The above massless BH's also carry minimal charges [of more than one U(1) field, but these still have to be diagonalized under supergravity]. It is also irresistible to compare black diholes with Cooper pairs in the BCS theory of superconductivity. In spite of the many differences the analogies are very appealing.

We cannot, however, ignore an important issue: How can supersymmetry be compatible with objects with negative mass? The ADM mass of a massless $\mathrm{BH}$ is zero, to start with, and there is no problem in admitting that the composite object could be supersymmetric. However, the unbroken supersymmetry of a composite object is 
the common sector of the unbroken supersymmetries of its components: the Killing spinor has to satisfy all the constraints that the presence of each component imposes (see, for instance, Refs. [13,14]). That is, the components have to admit Killing spinors themselves.

Now, there seems to be a problem with those constituents that have negative mass. Certainly, they cannot be supersymmetric: supersymmetry implies a positivity bound on the mass $[15,16]$. However, they can still admit Killing spinors (whose existence is necessary [17] but not a sufficient condition to have supersymmetry). In fact, it is easy to see by direct calculation that the $a=\sqrt{3}$ multiBH metrics (for instance) always admit Killing spinors for any choice of the harmonic function $V$ (see, for instance, Ref. [10]). [This may look strange to the reader that knows that Killing spinor techniques (Nester constructions) are used to prove the positivity of the mass and more restrictive bounds [18]. However, in all cases there are additional assumptions in the form of inequalities that the energy-momentum tensor has to satisfy. They are probably violated in the cases of negative mass.]

At the level of the supersymmetry algebra, the existence of Killing spinors means that certain supersymmetry charges annihilate the state. What does this mean for negative mass states? For an appropriate choice of the supersymmetry basis, the $N$ extended supersymmetry algebra can be written in this way [16]

$$
\left\{S_{\alpha( \pm)}^{m}, S_{\beta( \pm)}^{* n}\right\}=\delta_{\alpha \beta} \delta^{m n}\left(m \pm\left|z_{i}\right|\right),
$$

where $i=1, \ldots,[N / 2]$ and all other anticommutators vanish. Since the operators on the left hand side of these equations are positive, we have the bounds

$$
\begin{aligned}
& m-\left|z_{i}\right| \geq 0, \\
& m+\left|z_{i}\right| \geq 0 .
\end{aligned}
$$

Positive mass supersymmetric objects saturate one of the first bounds Eq. (16) and satisfy all the others. The saturation of one of the first bounds is associated to the existence of a supersymmetry charge that annihilates the corresponding state. That charge is associated to the Killing spinor. The rest of the charges act nontrivially and in a way consistent with the supersymmetry algebra on the state and their action on it generates (shortened) supermultiplets [17].

For a negative mass object admitting Killing spinors there must be a supersymmetry charge that annihilates the corresponding state. A supersymmetry bound of the second type Eq. (17) is saturated but all bounds of the first type are violated. (Observe that the quadratic form of the bounds, $m^{2}-\left|z_{i}\right|^{2} \geq 0$, which is enough to have an extreme solution of the equations of motion and Killing spinors, can be satisfied by negative mass objects.) Then, there are no other supersymmetry charges to complete the algebra; one cannot build supermultiplets and the state cannot be said supersymmetric.

In a bound state with a positive mass supersymmetric object, there can be compensations in the masses and charges and, if the total mass in not negative, since both components admit Killing spinors, the composite object can be supersymmetric.

Supersymmetry forbids the existence of isolated negative-mass objects, but it does not forbid their existence in non-negative mass bound states, just as quarks do not exist in isolation at low energies.

The author would like to thank Luis Álvarez-Gaumé, Renata Kallosh, and Jorge Russo for helpful discussions. He would also like to thank M. M. Fernández for her support.

*Electronic address: tomas@surya20.cern.ch

[1] A. Strominger, Nucl. Phys. B451, 96 (1995).

[2] K. Behrndt, Nucl. Phys. B455, 7137 (1995).

[3] R. Kallosh, Phys. Rev. D 52, 6020-6042 (1995).

[4] R. Kallosh and A. Linde, Phys. Rev. D 52, 7137-7145 (1995).

[5] R. Kallosh and A. Linde, Reports No. SU-ITP-95-26 and No. hep-th/9511115.

[6] T. Ortín, Phys. Rev. D 52, 3392-3405 (1995).

[7] M. Cvetič and D. Youm, Phys. Lett. B 359, 87-92 (1995).

[8] M. Cvetič and A.A. Tseytlin, Phys. Lett. B 366, 95 (1996); M. Cvetič and A.A. Tseytlin, Reports No. IASSNS-HEP-95-102 and No. hep-th/9512031.

[9] J. Rahmfeld, Reports No. CTP-TAMU-51/95 and No. hepth/9512089.

[10] R. R. Khuri and T. Ortín, Reports No. CERN-TH/95-348, Mc-Gill/95-62 and No. hep-th/9512177.

[11] J. Maharana and J.H. Schwarz, Nucl. Phys. B390, 3 (1993).

[12] B. R. Greene, D. R. Morrison, and A. Strominger, Nucl. Phys. B451, 109-120 (1995).

[13] T. Ortín, Phys. Rev. D 47, 3136-3143 (1993).

[14] T. Ortín, Phys. Rev. D 51, 790-794 (1995).

[15] E. Witten and D. Olive, Phys. Lett. 78B, 97 (1978).

[16] S. Ferrara, C. A. Savoy, and B. Zumino, Phys. Lett. 100B, 393 (1981).

[17] G. W. Gibbons, in Supersymmetry, Supergravity and Related Topics, edited by F. del Águila, J. de Azcárraga, and L. Ibáñez (World Scientific, Singapore, 1985), p. 147.

[18] G. W. Gibbons and C. M. Hull, Phys. Lett. 109B, 190 (1982). 